\title{
DEPTH STRUCTURE IN THE GAS IN SHAPLEY III ALONG THREE LINES OF SIGHT
}

\author{
DOMINIK J. BOMANS ${ }^{1}$, KLAAS S. DE BOER ${ }^{1}$, JAN KOORNNEEF 2 \\ 1 Sternwarte, University of Bonn, D-5300 Bonn, Germany \\ 2 ESA, Space Telescope Science Institute, Baltimore, USA
}

Shapley Constellation III in the Large Magellanic Cloud (LMC) is surrounded by a ring of HII regions, called Supershell LMC 4 (Meaburn 1980). In the direction of the centre of this spatial strucutre very little neutral interstellar gas is found (McGee \& Milton 1964). The spatial arrangement suggests that a hole exists in the gas layer of the LMC, one which may be blown out by the effect of the winds of the stars of Constellation III or by supernova blasts from the same area. Shock waves moving into surrounding neutral gas triggered star formation in the outer shell. Left inside would be a volume of diluted, and probably hot, ionized gas.

To probe the spatial structure of the gas we have collected high dispersion IUE spectra of some stars inside LMC 4. We have analysed the interstellar lines from the various elements and ions available and are able to derive the velocity structure of the lines of sight observed (Bomans \& de Boer 1989). We see a well-defined absorption component near $230 \mathrm{~km} \mathrm{~s}^{-1}$ and a broader structure around $280 \mathrm{~km} \mathrm{~s}^{-1}$. The column density of neutral gas is small, as confirmed from the HI $21-\mathrm{cm}$ profiles of Rohlfs et al. (1984). We have redetermined the spectral type of the stars from the IUE spectra in order to estimate the strength of the stellar Lyman-Alpha (L $\alpha)$ line. Two stars are of early enough type to allow the determination of $\mathrm{N}(\mathrm{H})$ from $\mathrm{L} \alpha$.

The dynamics of the gas of LMC 4 is unclear. There is a rather large spread in the velocities of the HI 21-cm components (Bomans et al. 1990), as derived from the Rohlfs et al. (1984) data. Our analysis does not lead unambiguously to the expanding shell structure as proposed by Dopita et al. (1985). Rather, the $21-\mathrm{cm}$ data indicate a chaotic velocity strcture.

From the metal absorption lines we can derive column densities of $\mathrm{Si}, \mathrm{S}, \mathrm{Fe}$, and $\mathrm{Zn}$ in the two gas components. Numbers are given in Table 1. The column densities are related to $S$, an element which does not deplete due to dust grains. The abundances found compare favourably with results for LMC gas from other studies (see de Boer 1990). The abundance ratio of $\mathrm{Zn}$ to $\mathrm{S}$ is close to the solar value. Si shows little depletion, in particular in the $230 \mathrm{~km} \mathrm{~s}^{-1}$ gas.

Table 1. Logarithmic relative abundances $\mathrm{X} / \mathrm{S}$ seen toward LMC 4

\begin{tabular}{|c|c|c|c|c|c|c|c|c|}
\hline \multirow[b]{2}{*}{$v(r a d)$} & \multicolumn{2}{|c|}{ Sk -67206} & \multicolumn{2}{|c|}{$\mathrm{Sk}-66118$} & \multicolumn{2}{|c|}{$S k-66 \quad 100$} & \multirow[t]{2}{*}{$\mathrm{R} 136$} & \multirow[t]{2}{*}{ Sun } \\
\hline & 230 & 280 & 230 & 280 & 230 & 280 & & \\
\hline $\begin{array}{l}\text { SiII } \\
\text { FeII } \\
\text { ZnII }\end{array}$ & $\begin{array}{l}-0.1 \\
-1.1 \\
-2.7\end{array}$ & $\begin{array}{l}-0.7 \\
-0.5 \\
-1.6:\end{array}$ & $\begin{array}{r}0.6 \\
-0.1 \\
>-2.5\end{array}$ & $\begin{array}{l}- \\
- \\
-\end{array}$ & $\begin{array}{r}0.2 \\
-0.9 \\
-\end{array}$ & $\begin{array}{l}-0.6 \\
-0.9 \\
-2.9:\end{array}$ & $\begin{array}{c}-0.4: \\
- \\
-3.2\end{array}$ & $\begin{array}{l}+0.3 \\
+0.3 \\
-2.6\end{array}$ \\
\hline$N(S I I)$ & 14.7 & 15.4 & 14.7 & - & 14.2: & 15.6 & & \\
\hline
\end{tabular}

R. Haynes and D. Milne (eds.), The Magellanic Clouds, 436-437.

(c) 1991 IAU. Printed in the Netherlands. 
The presence of absorption by CIV in the spectra of two stars (Sk -66 206 and Sk -66 100) at 250 $\mathrm{km} \mathrm{s}^{-1}$ is very interesting. The column density amounts to approximately $\log \mathrm{N}(\mathrm{CIV})>13$. If this represents gas in collisional ionization, and if one assumes for the $\mathrm{C}$ abundance the value found from emission lines, $\mathrm{C} / \mathrm{H}=-4.1$ (Dufour 1984), then this is equivalent to $\log \mathrm{N}(\mathrm{HII})>18$ of ionized gas. Since it is likely that the stars are in the rear of Constellation III and assuming that LMC 4 is spherical, the line of sight to the stars (both are somewhat to the side of LMC 4) would go through perhaps $500 \mathrm{pc}$ of ionized gas. Thus, one would find a density of $\mathrm{n}(\mathrm{H}+)>10^{-3} \mathrm{~cm}^{-3}$ and a pressure of $>100 \mathrm{~cm}^{-3} \mathrm{~K}$.

\section{References}

Bomans, D., de Boer, K.S. (1989), Recent Developments of Magellanic Cloud Research, K.S. de Boer, F. Spite, G. Stasinska (eds.) (Observatoire de Paris: Paris) p.137.

Bomans, D.J., Grebel, E.K., de Boer, K.S. (1990), Astron. Ges. Abs. Ser. 4, 78.

de Boer, K.S. (1990), these proceedings.

Dopita, M.A., Mathewson, D.S., Ford, V.L. (1985), Astrophys. J. 297, 599.

Dufour, R. (1984), IAU Symp 108 Structure and Evolution of the Magellanic Clouds, S. van den Bergh, K.S. de Boer (eds.) (Reidel: Dordrecht) p.353.

McGee, R.X., Milton, J.A. (1964), IAU Symp 20 The Galaxy and the Magellanic Clouds, F.J. Kerr, A.W. Rodgers (eds.) (Aust. Acad. Sci: Canberra) p.289.

Meaburn, J. (1980), M.N.R.A.S. 192, 365.

Rohlfs, K., Kreitschmann, J., Siegman, B.C., Feitzinger, J.V. (1984), Astron. Astrophys. 137, 343. 